\title{
Retrait-gonflement des sols argileux et des marnes
}

\section{J.-F. SERRATRICE}

Laboratoire régional des ponts et chaussées d'Aix-en-Provence CETE Méditertanée CS70499

13593 Aix-en-Provence Cedex 3

jean-francois.serratrice@ equipementgouv.fs
Cet article présente divers aspects expérimentaux du gonflement et du retrait des matériaux sensibles aux variations hydriques, d'après des essais de laboratoire. Les premiers exemples montrent les résultats de deux types d'essais oedométriques de gonflement : les essais de gonflement par paliers successifs et les essais de gonflement en parallèle. Les exemples suivants donnent tour à tour une illustration de la cinétique du gonflement, des effets tridimensionnels du gonflement, puis de la dégradation des propriétés mécariques consécutives au gonflement. Un autre exemple concerne les difficultés expérimentales tiées au contrôle des conditions hydriques pendant des essais de longue durée sur des matériaux sensibles aux variations de teneur en eau. Puis des exemples d'essais de retrait avec enregistrement en continu sont commentés. Enfin, une vision plus synthétique de l'amplitude des variations de volume de retrait/gonflement est proposée, basée en première approche sur la relation de cette a dynamique de retrait/ gonflement $w$ avec l'indice des vides initial du sol.
This paper presents various experimental aspects of swelling and shrinkage of sensitive soils to moisture changes, observed in laboratory tests. The fist examples show the results collected by two types of oedometric tests: swelling tests with successive stages and pardilel swelling tests. Next examples give successively some illustrations about the kinetic of swelling. the three-dimensional effects of swelling and the damage degradation produced on the mechanical properties of the soil as consequence of swelling. Another example concerns experimental difficultes arising with the control of moisture during long term tests on sensttive soils. Then shrinking tests with continuous measurement are presented. A more syothetic view is suggested on the amplitude of shrinking and sweiling volume changes, based on the dependence of this "shrinking swelling dynamic" to void ratio, as first estimate. 


\section{Introduction}

Les essais de laboratoire constituent des outils privilégiés pour mesurer les variations de volume de retrait et de gonflement des sols. De très nombreux travaux expérimentaux ont été consacrés à ces essais, en mettant en ceuvre des procédures diverses. Certaines de ces procédures sont normalisées. Les procédures courantes opèrent à une échelle macroscopicue sur des éprouvettes de dimensions centimétriques. Mais les processus de gonllement et de retrait mettent en jeu différents facteurs el différents phénomènes physiques qui prennent corps à une échelle microscopique et restent non directement accessibles à l'échelle des éprouvettes d'essais. Cela explique en partie la variété des protocoles d'essais et le caractère empirique de leur interprétation.

Cet article montre différents aspects du gonflement et du retrait pour des terrains de natures diverses comme des argiles ou des marnes testées dans Ieur état naturel, des sols compactés ou des sols déstructurés. Ces résultats ont été obtenus à l'occasion de programmęs d'essais de laboratoire effectués dans le cadre de reconnaissances géotechniques pour des projets. Les résultats d'essais de gonflement seront présentés tout d'abord, obtenus au moyen de deux procédures particulières : les essajs de gonflement par paliers successifs et les essais de gonflement en parallèle. Les deux procédures fournissent les paramètres caractérisant le potentiel de gonflement du sol. Mais ces procédures sont pénalisées par des problèmes pratiques, à commencer par la durée des paliers de gonflement ou l'hétérogénéttế des matériaux testés dans leur état naturel.

La mise en ceuvre de ces deux procédures d'essais permet d'examiner ensuite différents aspects du gonfliement à partir d'exemples. Le premier aspect concerne la cinétique du gonflement. Celle-cl peut être très lente, en présence de marnes compactes notamment, ce qui a pour conséquence d'augmenter la durée des essais. Le second aspect s'adresse aux elfets tridimensionnels du gonflement. La mise en évidence de ces effets s'effectue au moyen d'oedomètres $\mathrm{K}$ ou d'oedomètres à paroi souple. Ces instruments permettent de mesurer la contrainte radiale pendant le gonflement, sous différentes conditions de déformation radiale. Si l'observation de ces effets tridimensionnels est importante pour la détermination des propriétés du sol étudié et leur transposition au comportement des ouvrages, elle ne l'est pas moins pour expliquer en partie les divergences observées entre les procédures d’essais cle gonflement au laboratoire. Enfin, un troisième aspect du gonflement concerne la dégradation des propriétés de déformabilité des terrains sous l'effet du gonflement, ce qui suggère qu'une transformation du sol s'opère parfois pendant le processus, à l'échelle de temps des essais de laboratoire.

En guise de transition avec les mesures de retrait, un exemple particulier donne une illustration des difficultés expérimentales liées au contrôle des conditions hydriques pendant des essais de longue durée sur des matériaux sensibles aux variations de teneur en eau. Puis des exemples d'essais de retrait sont donnés sur diflérents matériaux. Une procédure particulièrè a été mise en ouvre, qui permet d'enregistrer la courbe de retrait du sol en continu pendant sa dessiccation. La méthode s'applique à des sols dans leur état naturel, des sols compactés ou des sols déstructurés.
Pour tenter de donner une vue synthétique des vartations de volume de retrait/gonflement, une récapitulation est proposée enfin, qui est basée sur un ensemble de données obtenues sur divers matériaux dans différents états, la plupart des matériaux étant testés dans leur état naturel. Ce rapprochement des données expérimentales suggère que, en première approche, les pressions de gonflement et, plus généralement, l'amplitude des variations de volume de retrait/ gonflement dêpendent de l'indice des vides intial du sol. Dans les essais de dessiccation ou d'imbibition, un sol argileux lầche développera préférentiellement des déformations de retrait, contraîrement à un sol argileux compact qui sera prédisposé à gonfler.

\section{2}

\section{Exemples d'essais de gonflement}

Deux procédures courantes d'essais de gonflement sont présentées ci-après au travers d'exemples obtenus sur des mames, Ces deux procédures s'inspirent des procédures utilisées en pratique et décrites dans la littérature ou normalisées (ISRM, 1989 ; Sridharan et a!., 1986 ; Serratrice et Soyez, 1996 ; Bigot et Zerhouni, 2000). Les essais sont réalisés à l'aide d'appareils oedométriques sur des ếprouvettes de diamètre initial $\mathrm{d}_{0}=60 \mathrm{~mm}$ et de hauteur initiale $\mathrm{h}_{0}=25 \mathrm{~mm}$. Les pro priétés physiques et les compositions minéralogiques des matériaux testés sont données dans les tableaux I et II. Toutes les données ne sont pas disponibles pour tous les matériaux testés. I apparaît néanmoins que les marnes possèdent une fraction argileuse à dominante smectique dans beaucoup de cas.

9.1

\section{Essais de gonflement par paliers successifs}

Ce premier exemple montre les résultats d'un essai de gonflement par paliers successif́s effectué à l’oedomètre sur une marne très gonflante (marne d'Esna, Egypte; Guillaume et al., 2001). Sur la figure 1a, ka courbe contrainte/déformation est đonnée dans le plan $\left(\lg \left[\sigma_{2}\right)\right.$, e), où $\sigma_{a}$ est la contrainte axiale en échelle logarithmique et e l'indice des vides. Un premier cycle de chargement/déchargement/rechargement par paliers est applicué à la mame dans son état naturel en 24 heures, jusqu'à une contrainte axiale $\sigma_{\mathrm{a}}=1780 \mathrm{kPa}$. Pendant le cycle, l'éprouvette repose sur des plaques poreuses sèches. Elle est mise en imbibition sous ce palier après stabilisation de la défomation, puis elle est déchargée par paliers successifs et elle est maintenue en imbibition jusqu"au palier final pour lequel $\sigma_{\mathrm{g}}=3 \mathrm{kPa}$ (poids du piston). La durée de chacun des paliers de déchargement sous imbibition est de plusieurs mois (durée totale de l'essai : 2 ans). La marne est très peu compressible dans son état naturel (voir le cycle préliminaire sur la figure 1a]. L'imbibition sous $1780 \mathrm{kPa}$ provoque un très fort gonflement et l'amplitude du gonflement sous ce premier palier ne trouve pas d'égal sous les paliers de déchargement suivants [non représentés ici). Du coup, l'unterprétation classique de l'essai, qui consiste à comparer la courbe de déchargement dans l'état naturel à la courbe de déchargement sous imbibition et à chercher l'intersection des deux tangentes à ces courbes, conduit à une pression de gonflement très supérieure à $5 \mathrm{MPa}$ (maximum de l'échelle des abscisses). 
Tamaul Propriétés physiques des matériaux testés.

\begin{tabular}{|c|c|c|c|c|c|c|c|c|}
\hline Matériau & Blac & $\frac{w_{\text {mina }}}{(\%)}$ & (kN $\left.\mathrm{kN}^{\mathrm{m}} \mathrm{m}^{3}\right)$ & $e_{\text {[xatin] }}$ & 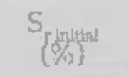 & $w_{\text {prigle }}$ & $w_{L}$ & $I_{I}$ \\
\hline Marne d'Esna & & 3.5 a 6.5 & 20.9 a 21.8 & $0,28 \mathrm{a} 0,33$ & 40 à 60 & 24 à 28,5 & 71 & 31 \\
\hline \multirow[t]{3}{*}{ Tunnel de Tartaiguilte } & 1 & 13 à 17 & 21,3 à 22,4 & 0,32 a 0,45 & 100 & 23 à 26 & 41 & 18 \\
\hline & 4 & 3,5 a 7,2 & 24,0 a 24,8 & 0,11 a 0,17 & 100 & 4,5 aे 9,3 & & \\
\hline & 5 & $8 \mathrm{z} 12$ & 21,6 a 22,9 & 0,27 à 0,42 & 100 & 18 尚 20 & & \\
\hline Tunnel d'Arbus & & $7.5 \mathrm{a} 21$ & 20,0 à 24,0 & 0,18 尚 0.64 & 100 & & & \\
\hline «Terres noires $\$$ & & $1 \mathrm{àg}$ & 23,0 à 27,0 & 0,05 a 0,27 & 100 & & & \\
\hline Oligocène Marseille & & 10,7 a 18,5 & $20,9322,8$ & 0,29 a 0,50 & 95 à 100 & 11,2 à 19,5 & 46 & 26 \\
\hline Mont Sior & & 3 当 20 & 20,7 当 25,2 & $0.09 \cdot 0.54$ & 100 & & 35 à 49 & 15 主 22 \\
\hline Chamoise & & 4 à 5 & 23,4 à 23,8 & 0,19 घे 0,22 & $(85)$ & 5 主 7 & & \\
\hline Bois du Peu & & 6 a 9 & 22,6 à 23,0 & 0,17 a 0,26 & 100 & 7,5 a 11 & & \\
\hline Les Milles & & $10 \mathrm{a} 28$ & 18,9 à 22,7 & 0,28 à 0,80 & 100 & 11 à 32 & 28 尚 38 & 9 当 14 \\
\hline LGV Est & & 14 à 18 & 20,7 当 21,6 & 0,40 a 0,51 & 100 & 18 à 20 & & \\
\hline LGV RR & & $23 \mathrm{a} 30$ & 18,6 à 19,7 & 0,65 à 0,85 & 100 & 26 à 30 & 48 à 51 & $16 \div 25$ \\
\hline Chavannes & & 6,5 a 6,7 & 21,7苗 21,3 & 0,30 a 0,32 & 56258 & 6,5 à 6,9 & & \\
\hline Limon de Goderville & & 13 aे 20 & 17, 1 当 20,1 & $0,58 \div 0,86$ & 45 a 91 & 95 à 100 & 41 & 19 \\
\hline Turin & & $10, \overline{7}$ aे 12,2 & 22,0 à 22,4 & 0,31 à 0,35 & 94 & 12,7 व 13,4 & & \\
\hline Béziers & & 1420 & 18,5 à 21,5 & 0,48 à 0,63 & $75 \geq 95$ & 17 à 25 & 30 aे 50 & 10 à 26 \\
\hline
\end{tabular}

Tabieau II Compositions minéralogiques des matériaux testés.

\begin{tabular}{|c|c|c|c|c|}
\hline Matériau & $\begin{array}{l}\text { Calcite } \\
\{\%\}\end{array}$ & $\begin{array}{l}\text { Quartz } \\
{[\%]}\end{array}$ & $\begin{array}{c}\text { Aryiles } \\
\text { (\%) }\end{array}$ & $\begin{array}{l}\text { argile dominante } \\
\text { et proportion dans la fraction angileuse }\end{array}$ \\
\hline Marne d'Esna & 20 & 5 & 75 & smectite $90 \%$ \\
\hline Tunnel de Tartaiguille & 25 & 45 & 30 & Snectite $45 \%$ \\
\hline Tunnel d'Arbus & 5 a 70 & 10 à 75 & 20 à 60 & smectite et interstratifiés smectiques 40 a $85 \%$ \\
\hline "Terres noires & 10 à 45 & 15 à 25 & 32 a 70 & interstratifiés illite smectite 70 a $80 \%$ \\
\hline "Terres noires $"$ & 10 à 45 & 15 à 25 & 32 à 70 & interstratifiés illite smectite 70 à 80 o. \\
\hline Oligocène Marseille & 21 à 34 & 10 à 15 & 55 à 70 & smectite 65 妾 $90 \%$ \\
\hline Tunnel du Mont Sion & 0 à 45 & $5 \geqq 47$ & 18 à 80 & smectite $35 \% 85 \%$ \\
\hline Tunnel de Chamoise & 45 & 20 & 35 & 䜣ite $45 \%$ \\
\hline LGV RR & 27 à $31^{*}$ & 6 à 14 & 59 à 63 & smectite 60 : $75 \%$ \\
\hline
\end{tabular}

* calcite et dolomie.

D'après la courbure observée sur le graphe $(\lg (\sigma), e)$, l'affaiblissement du gonflement pendant les paliers de déchargement sous imbibition ne permet pas de déterminer le potentiel de gonflement tel qu'il est exprimé traditionneliement, avec une déformation proportionnelle au logarithme de la contrainte axiale sur la totalité de la plage d'essai :

$$
e-e_{\mathrm{a}}=-\mathrm{K}_{\mathrm{s}} \lg \left(\sigma_{\mathrm{g}} / \sigma_{\mathrm{ag}}\right)
$$

oư $\sigma_{\mathrm{ag}}$ est la pression de gonflement, e l'indice des vides correspondant ( $\mathrm{e}=\mathrm{e}$ pour $\sigma_{\mathrm{a}}=\sigma_{\mathrm{a}, \mathrm{f}}$ ) et $\mathrm{K}$ le potentiel de gonflement exprimé en indice des vides, c'est à dire la pente des droites définies par l'équation (1) dans le plan ( $g\left(\sigma_{3}\right)$, e). D'autres essais ont montré que la réponse de la marne d'Esna dépend aussi du nombre de paliers de déchargement ef de la progression des contraintes entre les paliers. Bien souvent, les déformations ne sont pas totalement stabilisées sous un palier donné avant le passage au palier de déchargement suivant, en raison de la cinétique très lente du gonflement. Cette interférence entre les paliers nuit à l'interprétation de l'essai. Elle conduit à surestimen la pression de gonflement et sous-estimer le potentiel $\mathrm{K}_{\mathrm{e}}$.

\section{9}

\section{Essais de gonflement en parallèle}

Les essais de gonflement en parallèle sont effectués sur plusieurs éprouvettes simultanément. Après découpage dans uñ même échantillon, Jes éprouvettes sunl montées dans les cellules oedométriques sur des plaques poreuses sèches. Les celiules sont placées à leur tour sur les bâtis de chargement (Fig. 2). Un premier cycle de chargement-déchargement est appliqué sur chacune des éprouvettes dans leur état raturel jusqu’ả une contrainte axiale maximale $o_{\text {mag }}$ et sous des paliers de charge identiques. Puís les éprouvettes sont rechargées à des niveaux de contraintes différents et distribués en progression géométrique. Quand les défomations axiales des éprouvettes sont stablisées, les éprouvettes sont mises en imbibition avec de l'eau désaérée pendant une durée d'un mois et plus si nécessaire. La déformation axiale $\varepsilon_{\text {a }}$ est enregistrée pendant toute la durée de l'essai ( $\varepsilon_{\mathrm{a}}$ est le rapport de la variation de hauteur $\Delta \mathrm{h}$ à la hauteur initiale de l'éprouvette $\mathrm{b}_{0,}, \mathrm{\varepsilon}_{\mathrm{a}}=\Delta \mathrm{h} / \mathrm{h}, \Delta \mathrm{h}>0$ pour un tassement et $\Delta h<0$ pour un gonflement). 

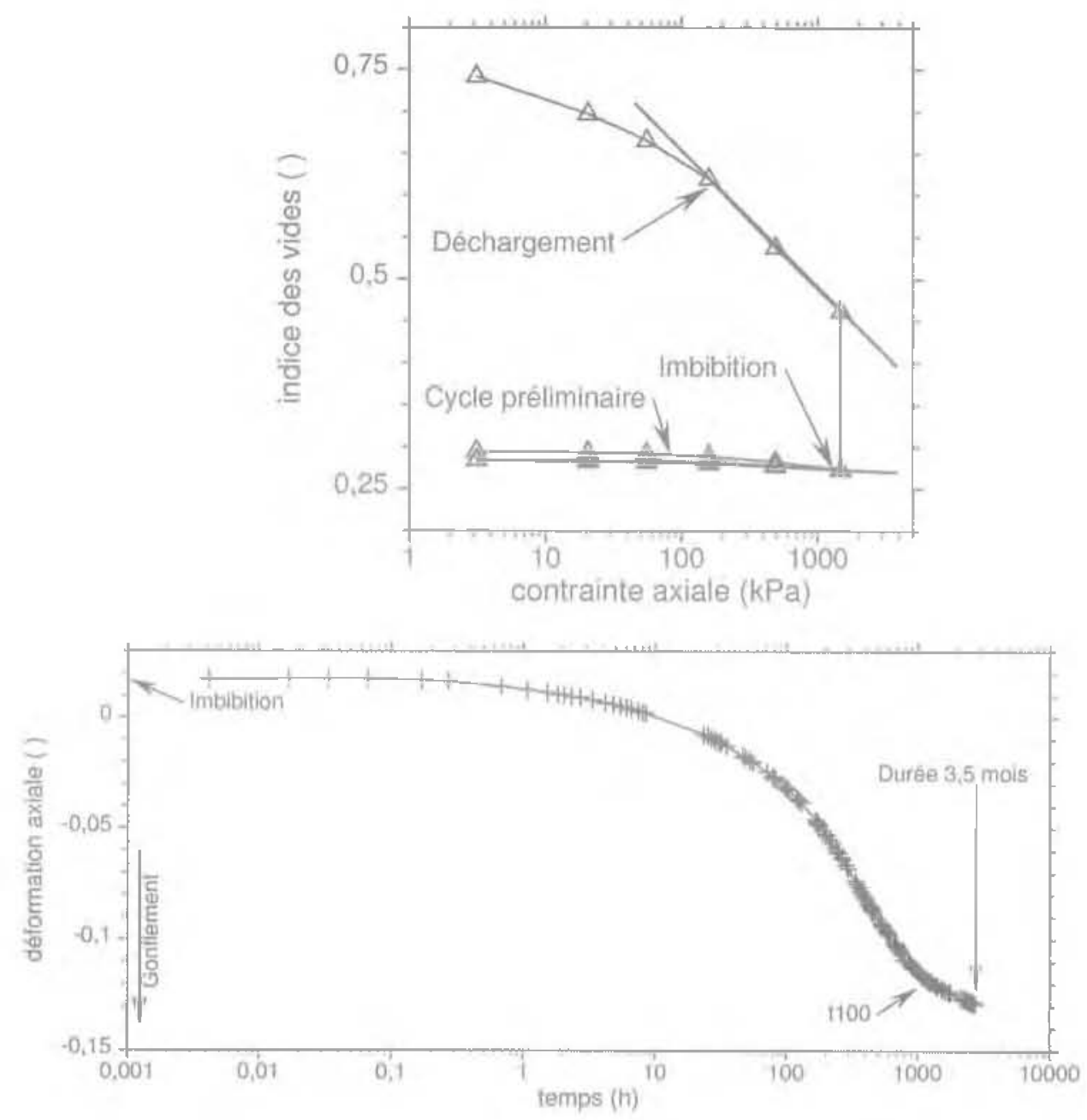

ค6. 1 Essai de gonflement par paliers successifs, marne d'Esna.

a) Courbe contrainte axiale/indice des vides; b) Courbe deformatin axiale/temps pendant le premier palier d'imbibition (1780 kPa).

Swelling test with successive stages, Esna marl.

a) Void ratio versus axial stress; b) Axial strain versus time during the first soaking stage (1,790 kPa).

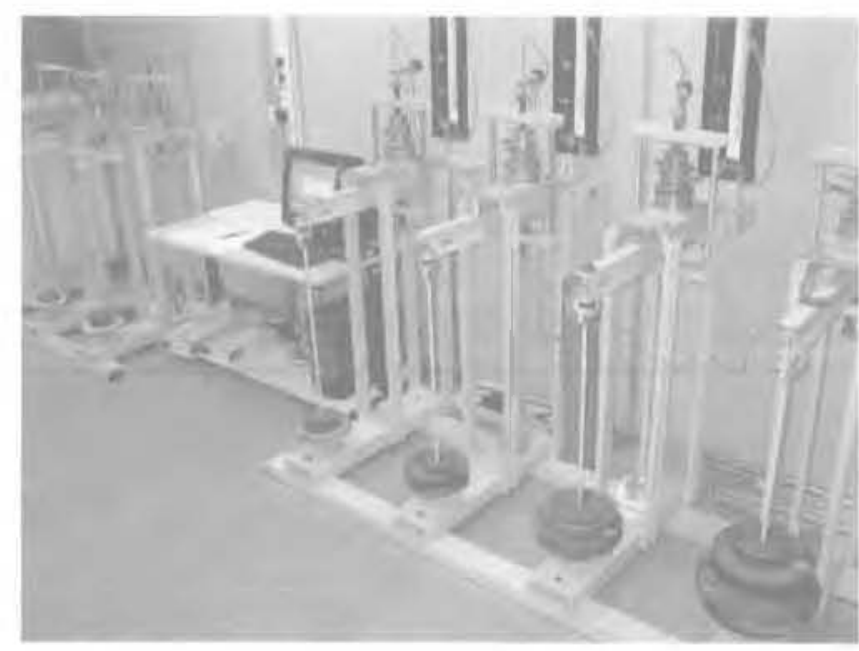

FG. \& Essaijs oedométriques en paralèle pour six éprouvettes. Bâtis de chargement. Chaîne de mesure.

Parallel swelling tests with six specimens. Loading frames. Data acquisition system.

La procédure des essais en parallèle présente l'avantage de rendre indépendantes les phases de gon- flement sous charge, par analogie avec te gonflement de différents éléments de volume plus ou moins confinés dans un massif naturel ou dans un remblai. II n'y a pas d'interférences entre les phases de gonflement, contrairement aux essais par paliers successifs. En contre partie, une hétérogénéité du terrain à l'échelle de l'échantillon concemé fait prendre le risque de tester des éprouvettes différentes, ce qui conduit à des difficullés d'interprétation de l'essai. Une méthode d'essai mettant en ceuvre plusieurs éprouvettes est définie dans la nomme NF P 94-091.

La figure $3 a$ montre les cycles de chargementdéchargement dans l'état naturel appliqués à quatre éprouvettes de mame tirées d"un même bloc (bloc 1) prélevés au front du tunnel dę Tartaiguille (Drôme). Les courbes sont reportées dans le plan $\left(\lg \left(\sigma_{3}\right)\right.$, ê. Après ce cycle préliminaire dans l'état naturel de la marne, les quatre éprouvettes sont chargées sous les contraintes axiales $\sigma_{a}=50,180,530$ et $1740 \mathrm{kPa}$ respectivement. Après stabilisation de la déformation axiale, les éprouvettes sont mises en irmbibilion en maintenant chacun des chargements. Les segments verticaux situés aux abscisses $50,180,530$ et $1740 \mathrm{kPa}$ représentent les gonflements observes des quatre éprouvettes respectivement, pendant ce premier palier d’imbibition. 

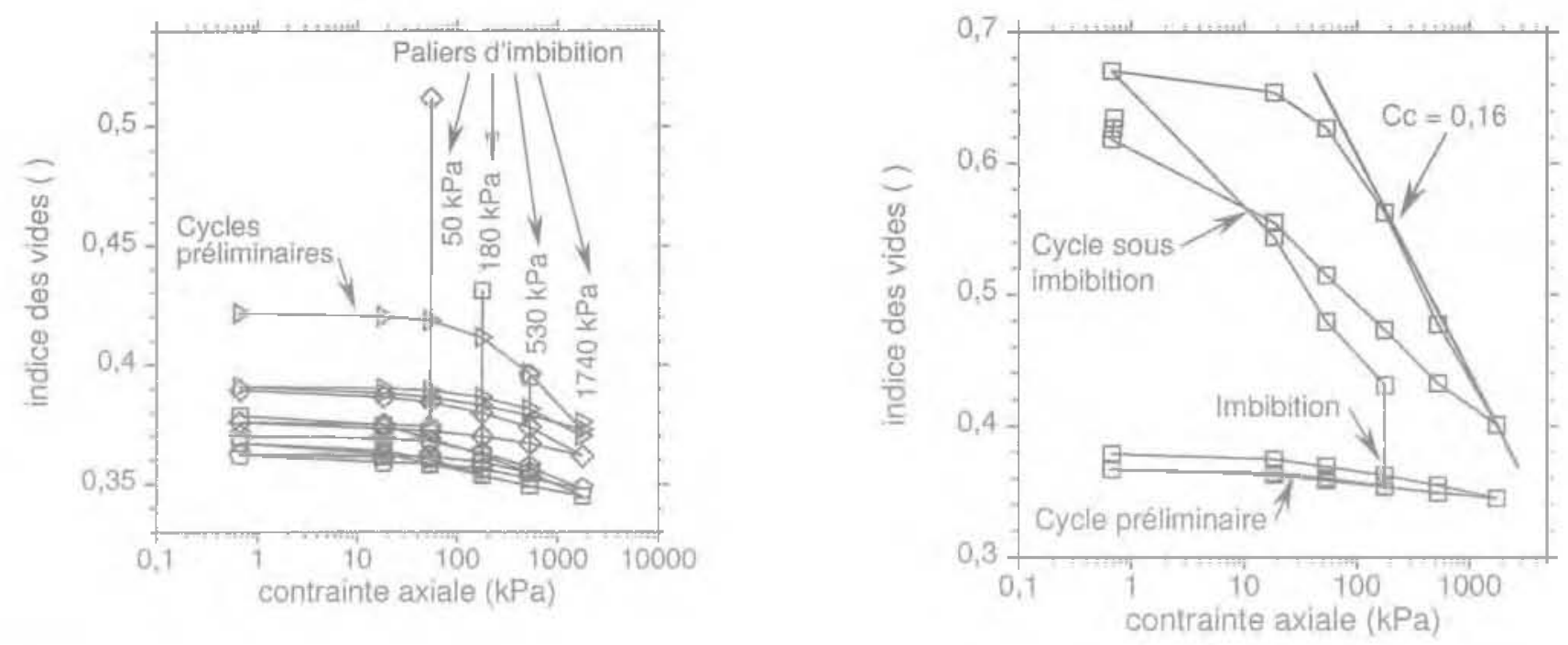

FG. 3 Essais de gonflement en parallele, marne de Tartaiguille (bloc 1).

a) Cycles préliminaires et paliers d'imbibition: b) Cycle supplérnentaire sous imbibition (iubibition sous $178 \mathrm{kPa}$.

Parallel swelling teses, Tartaiguille marl (block 1 ].

a) Preliminary cycles and soaking stages; b) Additional cycle under soaking (soaking under $178 \mathrm{kPa}$ ).

Bien souvent, la détermination du potentiel de gonflement des mames ou des argiles raides ne s'efrectue pas de façon aisée dans le plan ( $\lg \left(\sigma_{\mathrm{a}}\right)$, e), en raison de la variabilité de l'indice des vides initial qui est fréquemment observée à l'échelle des échantillons dans ces types de terrains plus ou moins indurés. Il est préférable alors d'exprimer le gonflement de chacune des éprouvettes par la variation de la déformation axiale $\Delta \varepsilon_{\mathrm{a}}$ pendant le palier d'imbibition en fonction du logarithme de la contrainte axiale sous la forme:

$$
\Delta \mathrm{E}_{\mathrm{a}}=\mathrm{K} \lg \left(\sigma_{\mathrm{a}} / \sigma_{\mathrm{ag}}\right)
$$

où $\mathrm{K}$ est le potentiel de gonflement exprimé en défor" mation $\left(K_{\mathrm{e}}=\left(1+\mathrm{e}_{0}\right) \mathrm{K}\right.$, $\mathrm{e}_{\mathrm{g}}$ indice des vides initial). La

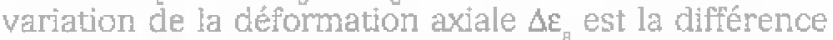
entre la déformation mesurée à la fin du palier d'imbibition et la déformation mesurée avant la mise en imbibition ( $\Delta \varepsilon_{\mathrm{a}}<0$ pour un gonflement).

L'interprétation de l'essai de la figure 3 sera donnée sur la figure5. Mals, au préalable, d'autres exemples de résultats d'essais de gonflement en parallèle sont donnés sur la fïgure 4. Il s'agit de cinq essais de gonflement réalisés sur différents échantillons de molasses du turnel d'Arbus (Hautes-Pyrênées), prélevés à différentes profondeurs dans plusieurs sondages carottés. Chaque essai comprend quatre éprouvettes découpées dans un même échantillon (sur une longueur de 30 cm environ). Les écarts entre les réponses $(0,008<\mathrm{K}<0,015$ et $650<\sigma_{3}<950 \mathrm{kPa}$ reflètent l'hétérogénéité des terrains à lechelle du site. Comme il est souvent observé, cette hétérogénéité transparaît à la fois par la variabilité de l'indice des vides, mais aussi par la variabilité de la composition minéralogique des molasses (et plus généralement des marnes), même sil la signature minéralogique de la formation est conservée globalement (voir le tableau II). I] est intéressant de noter que les contrainte maximales $\sigma_{\text {amax }}$ appliquées pendant les essais aboutissent à des gonflements quasi-nuls. Dans ce cas, les pressions de gonflement ont été dépassées par les chargements appliqués, ce qui conduit à une détermination plus süre de la pression de gonflement $\sigma_{a q}$

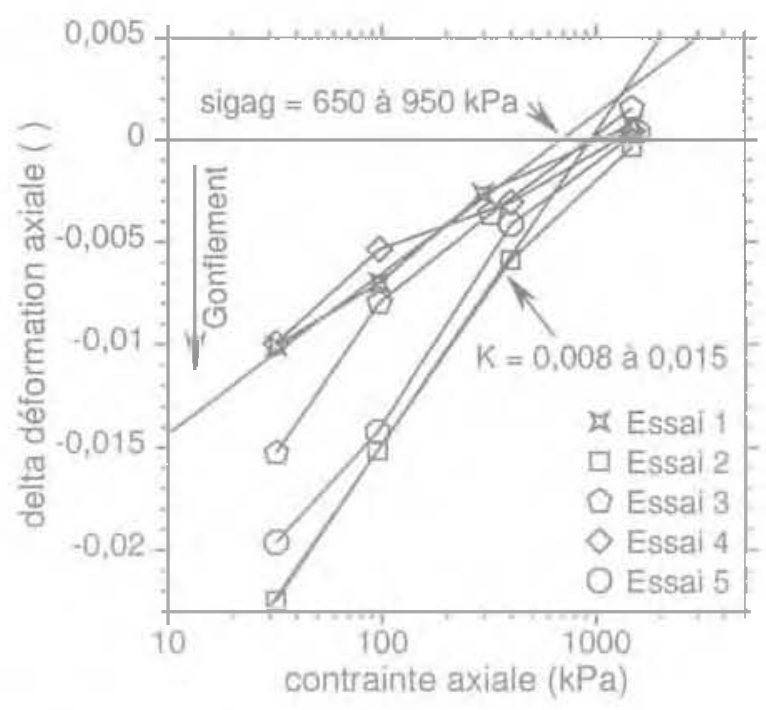

nE. Essais de gonflement en parallèle, molasses du site d'Arbus.

Parallel swelling tests, Artbus mar"'.

Quand la pression de gonflement du matériau testé s'avère ếlevée, il faut réaliser des essais oedométriques à haute pression, de façon à observer réellement l'an" nulation des déformations au-delà de $\sigma_{\text {aq. }}$. Tel est le cas de l'exemple de la figure 5, obtenu sur la marne du tunnel de Tartaiguille, cui montre une pression de gonflement égale à $\sigma_{a r}=2,1 \mathrm{MPa}$, pour des essais conduits jusqu'à $3,6 \mathrm{MPa}$ à l'aide d'un oedometre à haute pression. Le graphique montre trois essais en parallèle effectués sur différents blocs prélevés dans le mêrne front du tunnel. Dans cet exemple, une éprouvette supplémentaire est testée à haute pression et dans les mêmes conditions d'essaì que l'exemple de la figure 3 , ce qui porte à cinq le nombre d'éprouvettes découpées dans chacun des blocs 1 el 5. L’essai présenté sur la figure 3 a été réalisé dans le bloc 1. Les éprouvettes découpées dans le bloc 4 ne gonflent pas, car la marne est plus compacte, plus indurée et plus carbonatée que dans les autres biocs (voir le tableau I). 


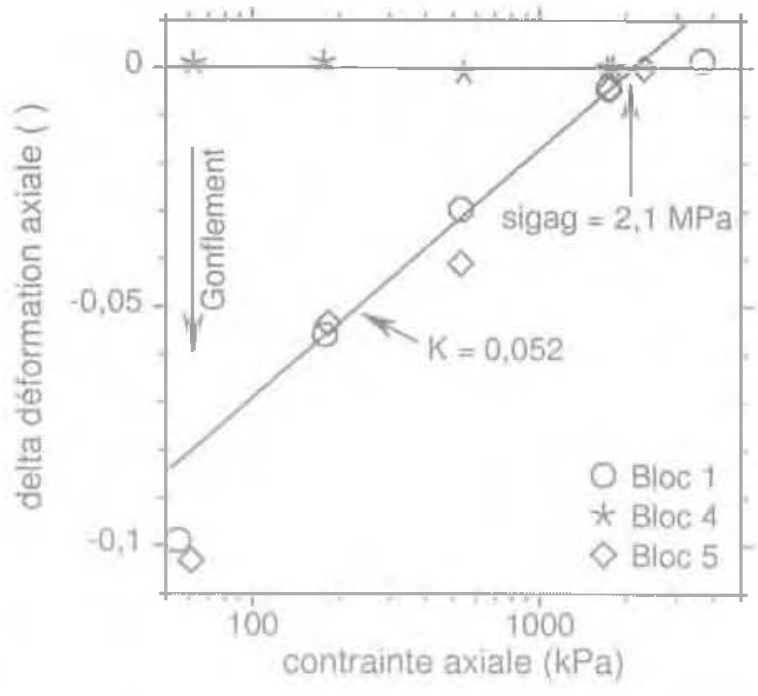

nG. 5 Essais de gonflement en parallẻle, marne de Tartaiguille (blocs 1, 4 et 5).

Parallel swelling tests. Tartaiguille mar' (blocks 1, 4 and 5).

Une variante des essais oedométriques en parallèle s'adresse aux sols compactés. Ces essaís de sensibilité des sols compactés aux variations de teneur en eau se pratiquent sur six éprouvettes densifiées par compactage semi-statique en un point d'état donné êt référencé par rapport aux caractéristiques Proctor (Mjeussens, 1993). Les essais oedométriques de sensibilité réalisés en parallèle s'avèrent parfaitement discriminants visà-vis de l'état initial du sol compacté et, plus généralement, de la nature du sol, quand on compere entre eux différents sols plus ou moins argileux ou un mêtme sol compacté à différents états (Serratrice, 1995, 2007).

\section{3}

\section{Aspects particuliers du gonflement en laboratoire}

\section{1}

\section{Cinétique du gonflement}

La figure 1b montre l'évolution de la déformation axiale en fonction du temps pendant le premier palier d'imbibition de la marne d"Esna, sous la contrainte axiale $\sigma_{a}=1780 \mathrm{kPa}$. Cette courbe est typique des réponses souvent observées en échelle logarithmique du temps, avec une forme de signe opposé à celuj des courbes de consolidation des argiles saturées. Une première phase s'apparente à la phase hydrodynamique de la consolidation, suivie par une phase cui, si la vitesse de déformation étaít positive (compression) serait considérée comme du fluage. Il s'agit en fait de gonflement sous charge constante (vitesse de déformation de signe négatif). La principale différence entre cette réponse et la consolidation des argiles réside dans la durée du processus, puisque le temps équivalent à la fin de consolidation ( $t_{100}$ ) mesurée ici avoisine 1000 heures (plus de 40 jours), pour une durée du palier égale à trois mois et demi.

Diverses interprétations ont été proposées dans la littérature pour caractériser cette cinétique du gonfle- ment, inspirées souvent du comportement observé sur les sols compactés non saturés. Les résultats prêsentés ici concernent des marnes testées dans leur état naturel, très compactes (indice des vides initial inférieur à 0,5, voire inférieur à 0,3 pour la marne d'Esna), préle vées à grande profondeur et saturées le plus souvent (tableaụ I]. Lanalogie de la cinétique du gonflement des marnes avec les courbes de consolidation des argiles saturées, qui est soulignée ici, tient en particulier à la représentation de la đéformation de gonflement dans une échelle logarithmique du temps. Mais il ne faut pas perdre de vie que, en matière de consolidation des argiles sałurées, cette représentation ne permet pas de séparer les deux mécanismes qui sont en jeu, le drainage de l'eau interstitielle et le fluage, pour produire la déformation différée du sol dans le temps (problème de l'origine du temps de fluage).

La figure $6 a$ montre les réponses à l'imbibition de quatre éprouvettes du site d'Arbus enregistrées pendant l'un des essais de gonflement en parallèle de la

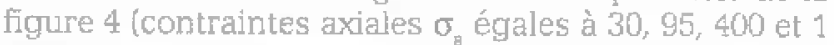
$565 \mathrm{kPa}$ respectivement; déformation axiale $\boldsymbol{E}_{\mathrm{s}}$ exprimée en fonction du logarithme du temps). L'éprouvette la plus chargée montre une déformation quasi-nulle á cette échelle du graphique. Pour les trois autres éprouvettes, l'amplitude finale des déformations croit à l'inverse de la charge. Les réponses présentent aussì une allure de courbes de consolidation a inversées $)$ avec des $t_{100}$ compris entre une et quatre heures. Il est intéressant de noter que le temps $\mathrm{t}_{100}$ croîl quand la charge diminue.

La figure 6b montre la première courbe du graphique précédent à une échelle dilatée ( $\left.\sigma_{\mathrm{a}}=1565 \mathrm{kPa}\right)$. Un gonflement apparait pendant la premiere heure d’imbibition, puis s'annule au profit d'un tassement pendant le reste de la durée de l'essai (un mois et demil. Des phénomènes de cette nature apparaissent parfois avec les sols argileux compactés. Sur le plan expérimental. l'une des difficultés rencontrées concerne la présence de plaques poreuses sèches pendant les cycles de chargement du sol dans son état naturel au début des essais. En pratique, la durée des paliers et leur nombre pendant ces phases préliminaires doit résulter d'un compromis entre le temps nécessaire pour assurer l'équilibre des charges à chaque étape du chargement et la réduction des effets de succion entre les plaques poreuses et le sol. L'exemple commenté ici donne une ilustration de ces difficultés expérimentales, le gonflement observé pendant la première heure d'imbibition pouvant résuiter du rééquilibrage de la succion apportée par les plaques poreuses pendant le cycle de chargement préliminaire. Quoiqu'il en soit, la pression de gonflement mesurée $\sigma_{\mathrm{a}}=680 \mathrm{kPa}$ est inférieure à la contrainte maximale appliquée $\left(\sigma_{\mathrm{H}}=1565 \mathrm{kPa}\right)$.

\section{2}

\section{Contrainte oedométrique radiale}

Un autre aspect important du gonflement conceme les elfets tridimensionnels. De nombreux essais de gonflement ont été effectués sur des argiles, des marnes ou des roches dans leur état naturel, ou des sols compactés, au moyen d'un oedomètre $\bar{K}$ à haute pression. L'intêrêt est' ici de mesurer la contrainte totale radiale $\sigma_{*}$ générée par l'imbibition. sous un palier de chargement axial donné. Ce type d'appareillage est utilisé plus conventionnellement pour déterminer le 

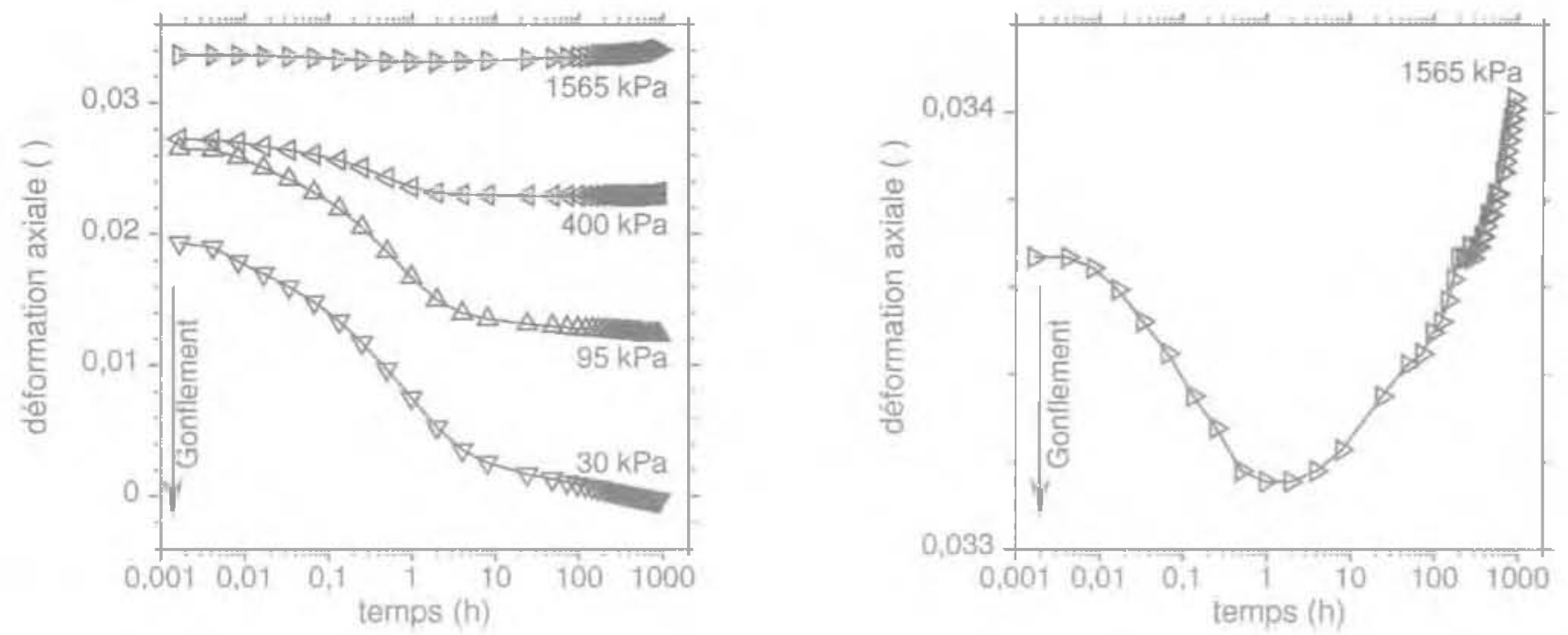

16. E Essajs de gonflement en parallèle, marne du site d'Arbus.

a) Déformations axiales pendant les paliers d'imbibition ; b) Deformation sous contrainte $\sigma_{\mathrm{a}}=1565 \mathrm{kPa}$.

Parallel swelling tests, Arbus marl.

a) Axat strains under soaking; b) Axal strain during the stage $\sigma_{z}=1,565 \mathrm{kPa}$.

chemin des contraintes oedométricues et son hystérésis pendant des cycles de chargements monotones drainés d'un sol ou d'une mame (Serratrice et Flavigny, 1996). Une éprouvette de diamètre $\mathrm{d}_{\mathrm{t}}=60 \mathrm{~mm}$ et de hauteur $\mathrm{h}_{\mathrm{o}}=25 \mathrm{~mm}$ est découpée à la trousse coupante et placée dans une bague oedométrique très peu déformable et équipée de jauges de déformation qui permettent de mesurer la contrainte radiale après étalonnage préalable de la bague. Le chargement est effectué à l'aide d'une presse, en condition drahée et à vitesse de déplacement constante. L'appareil permet d'appliquer une contrainte axiale maximale égale à $20 \mathrm{MPa}$ (Fig. 7). La réalisation de chargements par paliers est moins aisée, car le dispositif existant nécessite de contrôler la charge axiale manuellement.

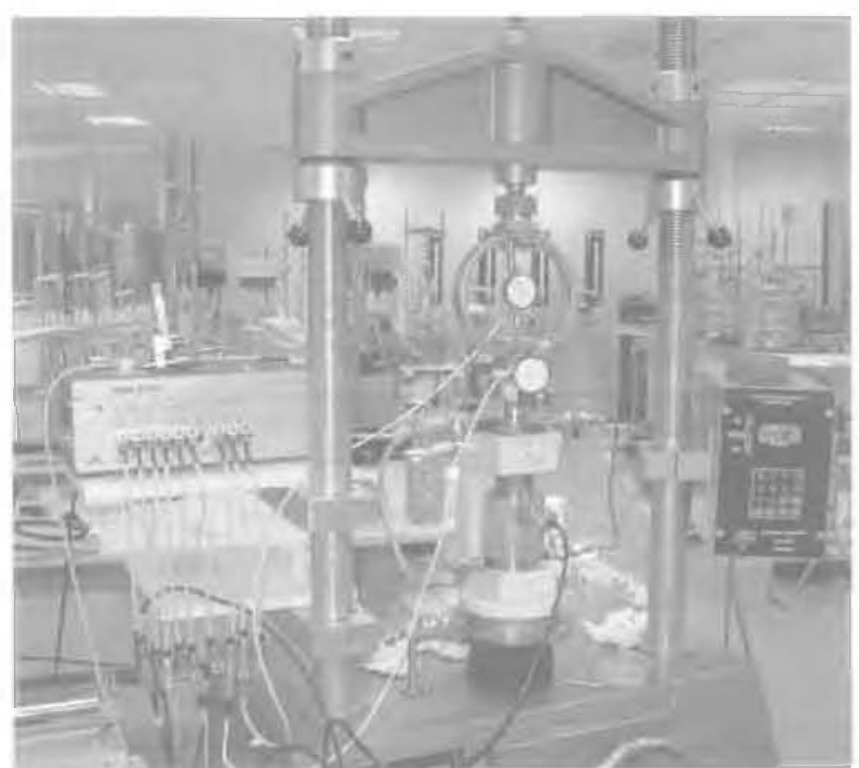

FG. 7 Essai te gonflement à Foedomètre $K_{\text {à }}$ haute pression. Appareil $K_{0}$, presse triaxiale et chaîne de mesure.

Swelling test with high pressure $\mathrm{K}_{0}$ oedometric ceil. $\mathrm{K}$ apparatus, loading frame and data acquisition systern.
La figure 8 montre la réponse de la marne d’Esna pendant un essai de gonflement réalisé avec un oedomètre $K$ à haute pression. La contrainte axiale est imposée initialement à $\sigma_{3}=1500 \mathrm{kPa}$ après un premier cycle de chargement dans l'état naturel de la marne et à l'aide d'une presse triaxiale (figure 8a). L'imbibition produit un gonflement $\left(\Delta \varepsilon_{\mathrm{a}}<0\right.$, figure $\left.8 \mathrm{~b}\right)$. Corrélativement, la contrainte radiale augmente pendant une centaine d'heures (figure 8a). La contrainte axiale augmente aussi, sous l'effet de la rigidité du bâti de chargement. Le rappel de la contrainte axiale à sa valeur imposée de $1,5 \mathrm{MPa}$ à plusieurs reprises (contrôle manuel) change peu la réponse radiale $\sigma_{r}$ qui demeure comprise entre 6,5 et 7,5 MPa. Dans le même temps, la déformation de gonflement $\Delta \varepsilon_{a}$ n'excède par $5 \%$. Après 700 heures d'essal, le déchargement total de l'éprouvette produit un très fort gonflement axial de près de $20 \%$ en plus de 200 heures. La contrainte radiale diminue aussi progressivement.

Dans un tel essai effectué en condition oedométrique, le gonflement de la marne produit une forte augmentation de la contrainte radiale, qui devient largement supérieure à la contrainte axiale. De ce fait, le chargement induit par le gonflement passe à un état de contrainte en extension caractérisê par un déviateur des contraintes $q=\sigma_{a}-\sigma_{r}$ négatif. Un tel état des contraintes peut conduire à la rupture de la marne en extension. Cette rupture est atteinte d'autant plus facilement que le gonflement a provoqué simultanément une dégradation des propriétés mécaniques du matériau par déstructuration (le sol se transforme au cours du gonflement).

Ces déformations de rupture en extension (déformations plastiques) se superposent aux déformations de gonflement, car ces deux types de déformations sont du même signe $\left(\Delta E_{\mathrm{a}}<0\right)$. En ce sens, les déformations de gonflement libre (charge axiale nulle) peuvent parfois se montrer excessivement surestimées à l'oedomètre, du fait de la superposition de déformations plastiques. La rupture dans loedomètre se manifeste alors de manière explicite avec l'apparition de plans de rupture sub-parallèles aux faces de l'éprouvette (disquage). Cette superposition des mécanismes de gonflement et de rupture s'observe ici au laboratoire. 

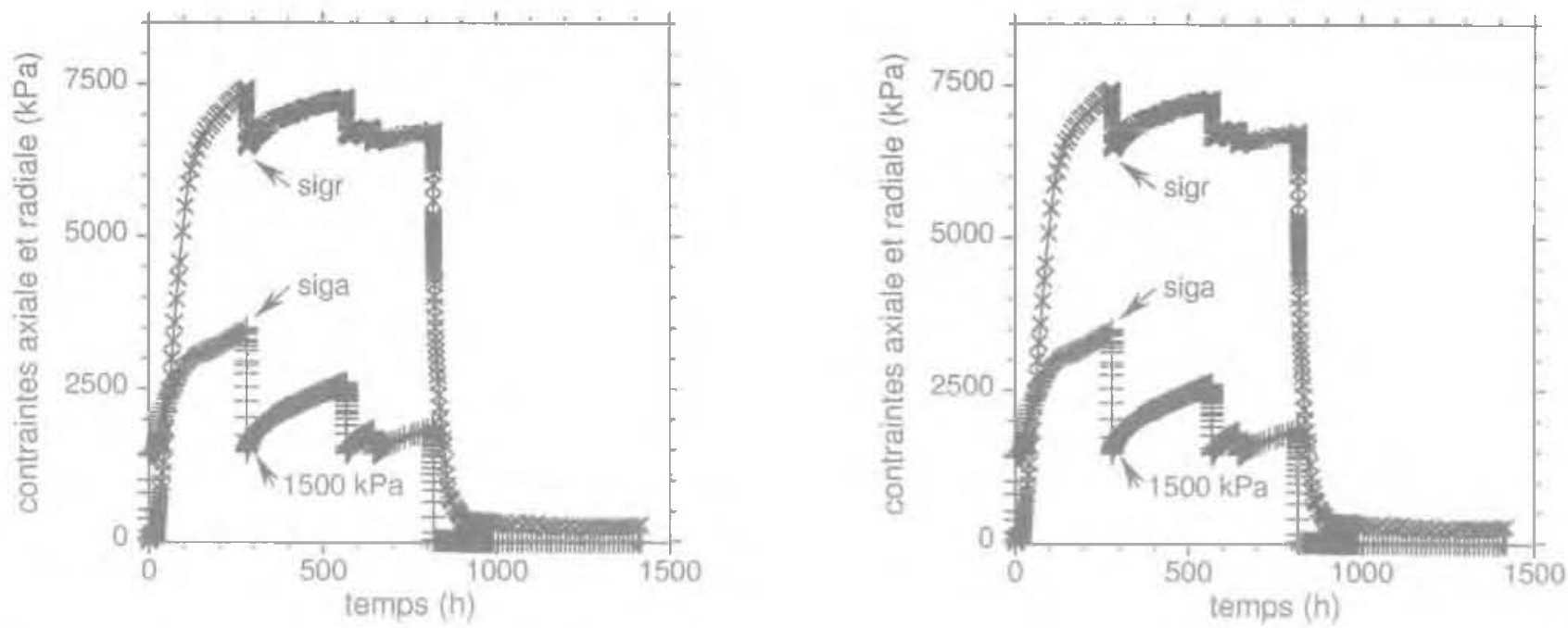

MG. 8 Essai de gonflement $K_{0}$, marme d'Esna.

a) Contraintes axiale et radiale en fonction du temps; b) Déformation axiale en fonction du temps.

$\mathrm{K}_{\mathrm{f}}$ swelling test, Esna marl

a) Axial and radial stresses versus time; b) Axial strain versus time

quand une contrainte axiale ne vient pas s'opposer al gonflement axial, à déformation radiale nulle. Cetté superposition peut apparaître aussi dans les ouvrages, en fond d'excavation notamment, ou en radier de tunnel, pour aboutir à un processus autoentretenu de fissuration du terrain et d'approfondissement progressif de la pénétration de l'eau au sein du massif, quand il n'est pas confiné.

La figure 9 donne un autre exemple d'essai de gonflement réalisé avec un oedometre $K_{n}$ à haute pression. Il s'agit d'une mame très compacte de l'oxfordien (w Terres noires \%, Hautes-Alpes). La contrainte axiale est maintenue à $1,9 \mathrm{MPa}$ pendant 35 jours. La pression radiale croît juscúà $\sigma_{r}=6,7 \mathrm{MPa}$ sans se stabiliser pendant la durée du palier.

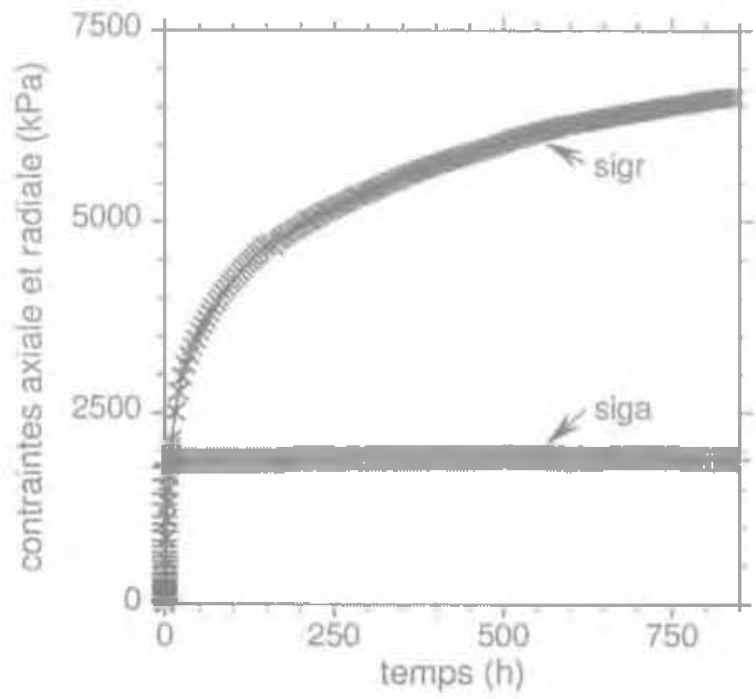

FG.9 Essai de gonflement $\mathrm{K}_{0}$, marne de Terres noires.

$K_{0}$ swelling test, Terres noires marl,

Le tableau III donne quelques valeurs de la contrainte radiale $\sigma_{\mathrm{s}}$ mesuré à l'oedomètre $\mathrm{K}_{\mathrm{o}}$ à haute pression sur différents matériaux au cours de séries d'essais comprenant plusieurs éprouvettes chargées dans la plage indiquée de la contrainte axiale $\sigma_{a}$ ( $e_{0}$ sont les indices des vides initiaux mesurés sur les différentes éprouvettes testées]. Des observations analogues ont été recuellies pour des sols argileux compactés, avec des pics du déviateur pendant la phase d'imbibition, puis de fortes amplitudes de gonflement en fin de déchargement. Les contraintes mesurées sont néanmoins plus petites sur les sols compactés en comparaison des terrains marneux plus ou moins indurés ou rocheux du tableau III. Les résultats d'essais de gonflement réalisés à l'oedomètre $\mathrm{K}$ sur une anbydrite du tunnel de Foix sont donnès à titre comparatif dans le tableau II, même si le gonflement ne trouve pas la mêre origine physique dans cette roche, en comparaison des marnes.

tABLEAU III Contraintes radiales $\sigma_{r}$ mesurées à l'oedomètre $\mathrm{K}$ à haute pression sur différents terrains, en fonction de la contrainte axiale appliquée $\sigma_{\mathrm{B}}$

\begin{tabular}{|c|c|c|c|c|}
\hline Site & Matériau & $\theta_{0}$ & $\stackrel{\sigma_{\mathrm{n}}}{\text { [MPa] }}$ & $\stackrel{\sigma}{\mathrm{MPa}^{\top}}$ \\
\hline $\begin{array}{l}\text { Tunnel } \\
\text { de Chamoise }\end{array}$ & mane & $0,13 \div 0,15$ & $0,1 \div 12,5$ & 3,8 a 13 \\
\hline Marne d'Esna & marne & 0,3 & 2 & 6,5 à 7,5 \\
\hline $\begin{array}{l}\text { Tunnel } \\
\text { de Tartaiguille }\end{array}$ & marne & 0,12 à 0,35 & $2 \mathrm{a} 7$ & 1,6 a 6,5 \\
\hline $\begin{array}{l}\text { “Terres } \\
\text { noires } \\
\text { Hautes-Alpes }\end{array}$ & martse & $0,08 \mathrm{a} 0,15$ & 2 & 1 à 7 \\
\hline $\begin{array}{l}\text { Tunnel du } \\
\text { mont Sion }\end{array}$ & molesses & 0,1 à 0,25 & 3 à 8 & 4 \\
\hline Tunnel de Foix & anhydrile & $(0,05)$ & 2,5 & 10 \\
\hline
\end{tabular}

Une approche expérimentale différente a été proposée par Windal (2001) et Windal et al. (2002) pour aborder les effets tridimensionnels du gonflement. Des oedomètres à paroi souple ont été mis au point et utilisés sur divers matériaux. Les essais sont de type oedo- 
métrique à chargement par palier. Des éprouvettes sont découpées dans un même échantillon et sont placées dans des bagues minces de différentes rigidités. Ces bagues mesurent la contrainte radiale $\sigma_{\mathrm{r}}$ et autorisent une déformation radiale proportionnelle à cette contrainte. Chaque essai se déroule par des paliers de déchargement sous imbibition.

La figure 10 montre les déformations axiales $\varepsilon_{\mathrm{a}}$ et Ies contraintes radiales $\sigma$ mesurées sur trois éprouvettes de marne de l'Oligocène (Marseille) pendant le premier palier d'imbibition sous une contrainte axiale $\sigma_{\mathrm{f}}=780 \mathrm{kPa}$. Les modules de rigidité des anneaux flexibles sont $\mathrm{K}_{\text {nn }}=370$, 580 et $3045 \mathrm{MPa}$ respectivement. Il apparait clairement que la contrainte radiale o est d'autant plus grande que la bague ( oedométrique » est rigide et que, dans le même temps, la déformation axiale $\varepsilon_{\text {s }}$ est plus grande. L'autorisation d'une déformation laterale induit donc une diminution concomitante de la pression de gonflement latérale et de la défor. mation axiale de gonflement. La pression radiale et la déformation axiale de gonflement sont maximales dans les conditions oedométriques « stricte » des essais $\mathrm{K}$, sans déformation latérale.

Sur le plan pratique, cette l'illustration de la mesure des cortraintes radiales pendant le gonflement iraduit une forte interaction entre l'éprouvette et la bague oedométrique, qui n'est pas sans conséquence sur les résultats de l'essai. Cela suggère que les gonflements mesurés dépendent du protocole d'essai et, en particulier, du niveau de contrainte axiale sous lequel est intié le gonflement pendant le premier palier d'imbibition. D'où les divergences observées entre les procédures courantes, divergences qui peuvent être accentuées par les imperfections des instruments de mesure (frottement du sol sur la bague oedométrique par exemple). l'évolution du sol pendant le gonflement, les interférences entre Ies paliers de déchargement sous imbibłtion, etc.

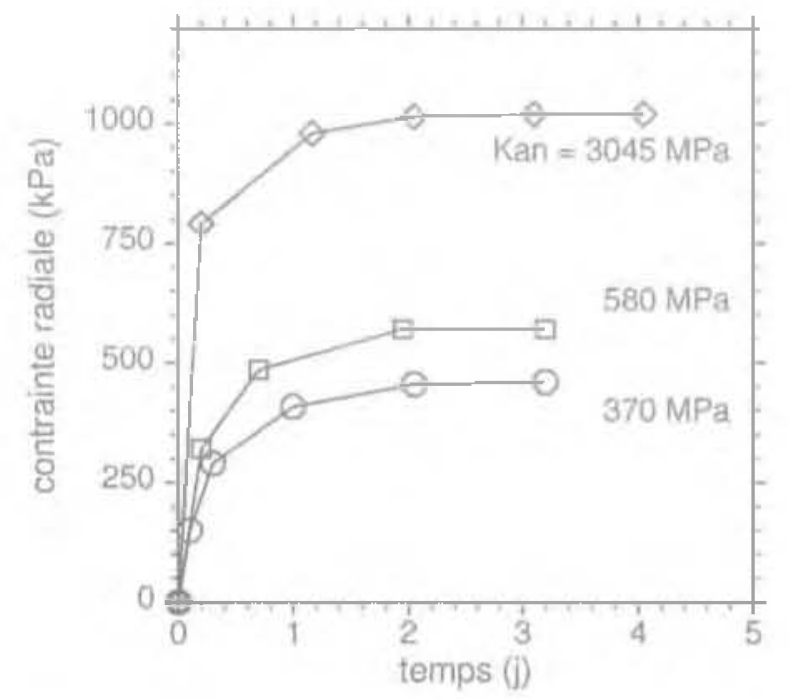

\section{Gonflement et déstructuration}

Le point suivant concerne l'évolution des terrains gonflants dı fait de l'imbibition, à l'échelle de temps des essais de laboratoire. Cette question a déjà été évoquée plus haut. L'exemple de la figure $3 \mathrm{~b}$ concerne la marne du tunnel de Tartaiguille et l'une des éprouvettes de la figure 3a léprouvette mise en imbibition sous $178 \mathrm{kPa}$ ). Ces essais ont été poursuivis après la première phase d'imbibition par un cycle de déchargement, rechargement, déchargement par paliers, sous imbibition. La courbe contrainte déformation de l'éprouvette soumise à imbibition sous la contrainte axiale $\sigma_{\mathrm{a}}=178 \mathrm{kPa}$ est représentée dans le plan $\left(\lg \left(\sigma_{\mathrm{a}}\right)\right.$, e), I] apparaît nettement que la compressibilité de la marne mesurée après le gonflement a largement augmenté en comparaison de sa compressibilité d'origine. Les trois autres éprouvettes ont montré un comporternent identque. Dotée d'un module d'Young de plusieurs GPa dans son état naturel, d'eprès des mesures réalisées en compression uniaxiale ou au triaxial à haute pression, la marne se retrouve après gonflement avec un coefficient de compressibilité $C_{c}=\Delta \mathrm{e} / \Delta \lg \left(\sigma_{s}\right)$ égal à 0,16 , c'est-à-dire un taux de compressibilité typique de celui d'une argile. Les déformations de gonflement s'avèrent presque totalement irréversibles par rapport à l'état naturel de la marne. Cette évolution peut être reliée vraisemblablement à l'évolution des minéraux argíleux sous l'effet de l'hydratation dans les conditions mécaniques particulières de l'essai, conjointement à des mécanismes d'interaction avec l'appareil d'essèi (fort état de contrainte d'extension). On peut parler alors de mécanisme d'altération à l'échelle de temps de l'essaj et, pour les ouvrages, d’altération à une échelle de temps humaine. Les sols se transforment du fait du gonflenent (Serratrice et Soyez, 1996).

Un exemple opposé est donnê sur la figure 11 où les pressions de gonflenent mesurées sur les molasses

1. 16 Essais de gonflement à l'oedomètre à paroi souple, marme de l'Oligocène. Paliers d'imbibition sous $\sigma_{\mathrm{E}}=780 \mathrm{kPa}$, $K_{\text {ar }}$ rigidité đes bagues.

a) Contraintes radiales en fonction du temps; b) Déformations axiales en fonction du temps (d'après Windal et al., 2002).

Swelling tests with flexible oedometers, Olgocene marl. Soaking stages under $\sigma_{\mathrm{a}}=780 \mathrm{kPa}, \mathrm{K}$, ring stifiness. a) Fadial strain versus time; b) Axial strain versus time (after Windal et al, 2002). 
du site du mont Sion (Haute-Savoje) sont reportées en fonction de leurs indices des vides initiaux (Gaudin et SerTatrice, 1998). La dispersion des indices des vides initiaux tient à la variabilité spatiale de la compacité des molasses, quỉ sont prélevées dans différents sondages carottés profonds. Des pressions de gonflement maximales apparaissent pour les indices des vides voisins de $e_{0}=0,15$, par opposition aux terrains plus compacts ou plus lâches $\left(0,09<\mathrm{e}_{0}<0,31\right)$. Cet exemple suggère la présence d'effets de structure plus ou rnoins marqués qui s'opposent à la déstructuration du matériau pendant ie gonflement. Ces effets sont modulés par la compacité du terraỉ comme exprimé ici, mais peuvent l'être aussi par la vartabilité de la composition minéralogique des molasses dans leur état naturel (tableaul II). Leroux (1972), puis Pejon et al. [1997), suggèrent que la composition minéralogique globale des marnes ne permet pas de caractériser seule le comportement de ces matériaux en présence d'eau et que leur texture ou leur micro-fissuration jouent un rôle prépondérant pendant le gonflement.

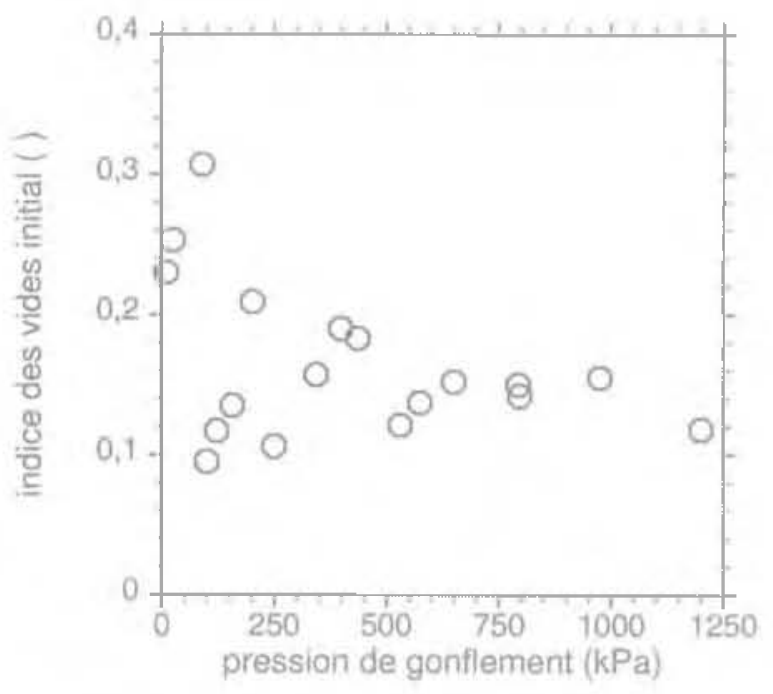

Fc. 11 Pressions de gonflement en fonction de I'indice des vides initial, molasses du site du mont Sion.

Swelligg pressure versus initial void ratio, Mont Sion marl.

\section{Problèmes posés par les essais de fluage sur les marnes}

Cet exemple vient en marge du sujet abordé ici, mais il permet de souligner les difficultés expérimentales qui sont rencontrées au laboratoire en matière de gonflement et de retrait. Il s'agit d'essais de fluage destinés à mesurer des vitesses de déformation sous contraintes constantes dans le cadre d'un projet de tunnel. La marne est très compacte, avec des indices des vides voisins de 0,1, et possède des modules d'Young compris entre 2 et 12 GPa et des résistances en compression uniaxiale comprises entre 3 et $50 \mathrm{MPa}$. La figure 12 montre les déformations axiales $\varepsilon_{8}$ enregistrées en fonction du temps pour quatre essais de fluage en compression uniaxiale. Pour être ísolées des conditions ambiantes, les éprouvettes de marne sont placées dans des cellules triaxiales sans fluide de confinement et sont protégées par deux membranes en latex grais. sées, avant d'être chargées. Dans un premier temps, les quatre éprouvettes sont soumises à des contraintes axiales égales à $674,1303,1936$ et $2568 \mathrm{kPa}$ pendant près de 200 heures, puis à des contraintes égales à 293, 545, 2568 et $1050 \mathrm{kPa}$ respectivement, pendant 600 heures supplémentaires (trois éprouvettes sont donc déchargées). Ces contraintes sont tès inférieures à la résistance de la marne. Les enregistrements montrent des déformations de compression, qui progressent indépendamment des charges appliquées et des déchargements. Pour tenter de confirmer cette évolution et au bout de 1000 heures, quelques gouttes d'eau ont été introduites au fond des cellules triaxiales sans contact avec les éprouvettes, ce qui a produit une inversion immédiate du sens des déformations. Les déformations du début de l'essai, qui pouvalent être interprétées comme du fluage, s'apparentent plus a des déformations de retrait du fait de leur inversion en gonflement quand les conditions hydriques sont modifiées dans les cellules triaxiales. Cet exemple montre les difficultés expérimentales qui sont rencontrées en matière de caractérisation mécanique des matériaux marneux au laboratoire sur des longues durées, guand les terrains sont sensibles aux variations des conditions hydriques.

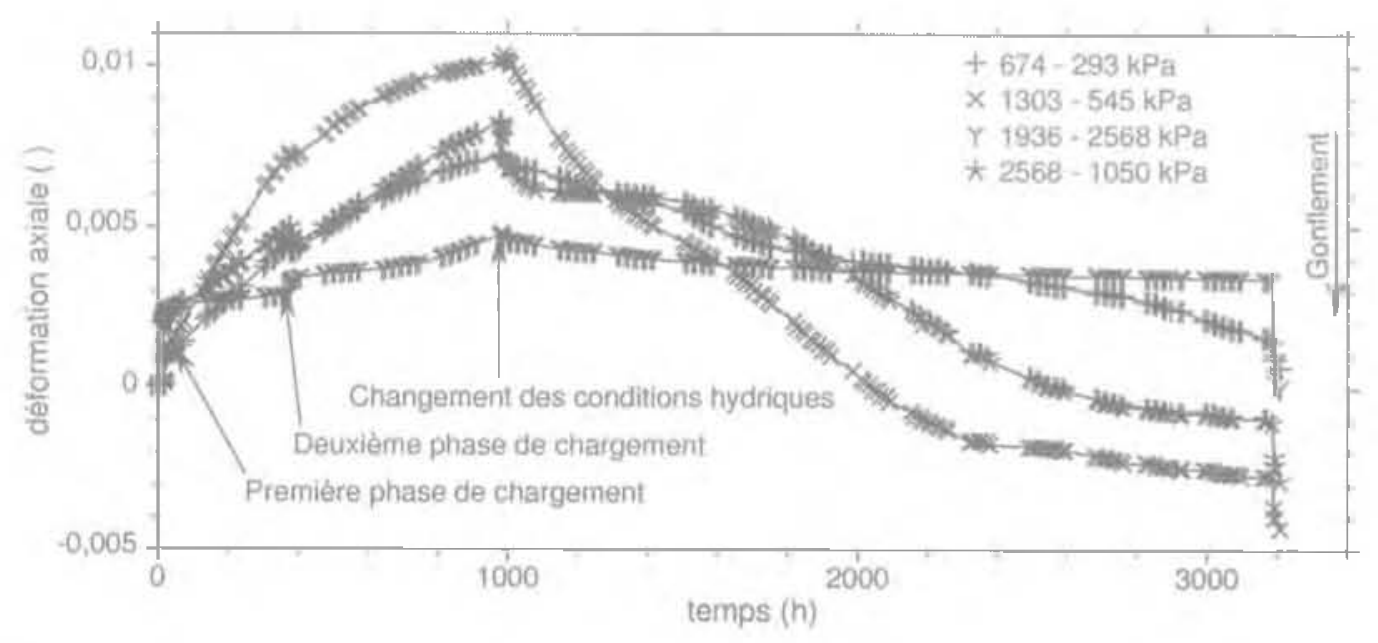

Fia. 1n Essais de fluage incontrôlés sue une marne. Déformation en fonction du temps. Uncontrolted creep tests on a marl. Axial deformations versus time. 


\section{Essais de retrait}

Pour un matériau non remanié, la limite de retrait est définie comme la teneur en eau pondérale aum dessous de laquelle le matériau ne présente plus de variation géométrique notable au cours de sa dessiccation (norme NF P 94-060-2). L'essai consiste à mesurer simultanément la déformation axiale et la teneur en eau d'une éprouvette à différents stades de sa dessiccation en atmosphere ambiante. Une varjante de cet essail a été mise au point afin de suive le retrait en continu (Serratrice, 2007). L'éprouvette est découpée à la trousse coupante. Léprouvette, la trousse et un capteur de déplacement placé dans l'axe de la trousse sont posés sur le plateau d'une balance de précision. L'enregistrement de la variation de la masse de l'éprouvette à l'aide de la balance et de sa variation de hauteur à l'aide du capteur de déplacement axial permet de suivre la courbe de retrait en continu. La déformation radiale n'est pas mesurée.

Des exemples d'essais sont donnés sur la figure 13 où sont reportées les courbes ( $w$, e), donnant l'indice des vides en fonction de la teneur en eau, obtenues sur une marne, une argile, un limon compacté et un limon déstructuré saturé. La droite de saturation d'équation $\mathrm{e}=\mathrm{w} \rho_{\mathrm{s}} / S_{\mathrm{r}} \mathrm{p}_{\mathrm{w}}$ est représentée sur le graphique l $\rho_{\mathrm{s}}$ masse volumique des grains solides, $\rho_{w}$ masse volumique de l'eau, $S_{\mathrm{r}}$ degré de saturation; ici $S_{r}=1$ et $\left.p_{s} / \rho_{w}=2,7\right)$. La mame ne montre pas de variation de volume à cette échelle du graphique, contrairement au limon remanié à une teneur en eau initiale de $58 \%$. L'argile présente une courbe de retrajt typique sur laquelle les caractéristiques de retrait, définies par les pentes des branches représentatives des variations de volume et les coordonnées du point d'intersection, sont asément identifiables. La courbe du limon compacté présente une allure comparable, mais le sol n’est pas saturé au départ.

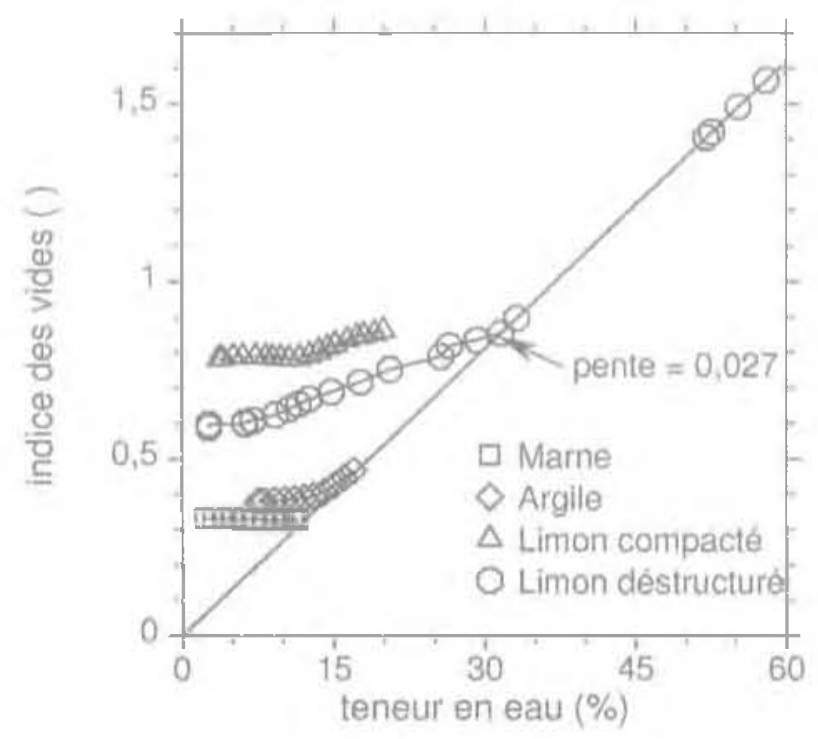

F6. 13 Essais de retrait avec enregistrement continu sur différents sols.

Shrinkage tests with continuous measurement on various soils.
Ces exemples montrent une large étendue des variations de volume de retrait observées sur les sols. Les expériences accumulées au fil du temps révèlent que la limite de retrait n'est pas une grandeur intrinsèque du sol. L'amplitude des déformations de retrait, d'une part, et la limite de retrait, d'autre part, dépendent de l'état initial du matériau et, pour les sols reconstitués ou compactés, de la méthode de préparation au laboratoire. Sur un plan pratique, l'une des difficultés soulevées par les essais de retrait provient de l'impossibilité d'opérer un contrôle mécanique de la déformation pendant la dessiccation en atmosphère ambiante, contrairement aux essais de gonflement.

\section{5}

\section{Variations de volume de retrait et de gonflement}

\section{1}

\section{Compacité et pression de gonflement}

La figure 14 montre les pressions axiales de gonflement $\sigma_{\text {ug }}$ et le potentiel de gonflement mesurés au moyen d'essais oedométriques sur différentes marnes. Comme précếdemment, les indices des vides sont reportés suivant l'axe des ordonnées du graphique. En abscisse, les pressions de gonflement $\sigma_{\text {ag }}$ sont graduées selon une échelle arithmétique. En chacun des points, l'amplitude du gonflement est représentée par un segment vertical d'abscisse $\sigma_{\mathrm{ag}}$ et de longueur $\Delta \mathrm{e}_{\mathrm{k}}$ (un double figuré représente les extrémités de chacun des segments verticatix) tel que

$$
\Delta \mathrm{e}_{\mathrm{q0}}=-\mathrm{K}_{\mathrm{v}} \lg \left(10 / \sigma_{\mathrm{ag}}\right)=\left(1+\mathrm{e}_{\mathrm{o}}\right) \mathrm{K} \lg \left(10 / \sigma_{\text {ag }}\right)
$$

c'est à dire l'augmentation de l'indice des vides obtenue entre une pression de référence égale à $\sigma_{a}=10 \mathrm{kPa}$ et la pression de gonflement. Cet indíce $\Delta e_{10}$ combine à la fois les paramètres $K_{\varepsilon}$ (ou $K$ ) et $\sigma_{a p}$. A l'échelle des ordonnées du graphique, qui exprime la grande variété des indices des vides initiaux des matériaux testés dans leur état naturel, y compris dans un mềme site, les segments verticaux sont réduits et peu visibles (double figuré d'abscisse $\sigma_{a j g}$ ).

Les points expérimentaux peuvent être regroupés en familles pour traduire une transition entre les terrains peu compacts associés à des faibles pressions de gonflement vers des terrains très compact associés à de fortes pressions de gonflement. Dans chacune des familles la dispersion des points experimentaux tient à la variété des compacités qui caractérisent le site de prélèvement. En chaque point, l'amplitude des gonflements $\Delta \mathrm{e}_{10}$ reste faible en regard de la variété des indices des vides initiaux (entre 0,07 et 0,8 sur ces exemples). De ce fait, tous les matériaux naturels gonflants présentent des pressions de gonflement plus ou moins grandes suivant leur nature et leur structure, mais les pressions les plus fortes ne peuvent être trouvées que parmi les terrains les plus compacts. Ce commentaire ne peul être détaché du fail que l'observation du gonflement s'effectue ici à une échellc macroscopique au moyen d'essais oedométriques. 


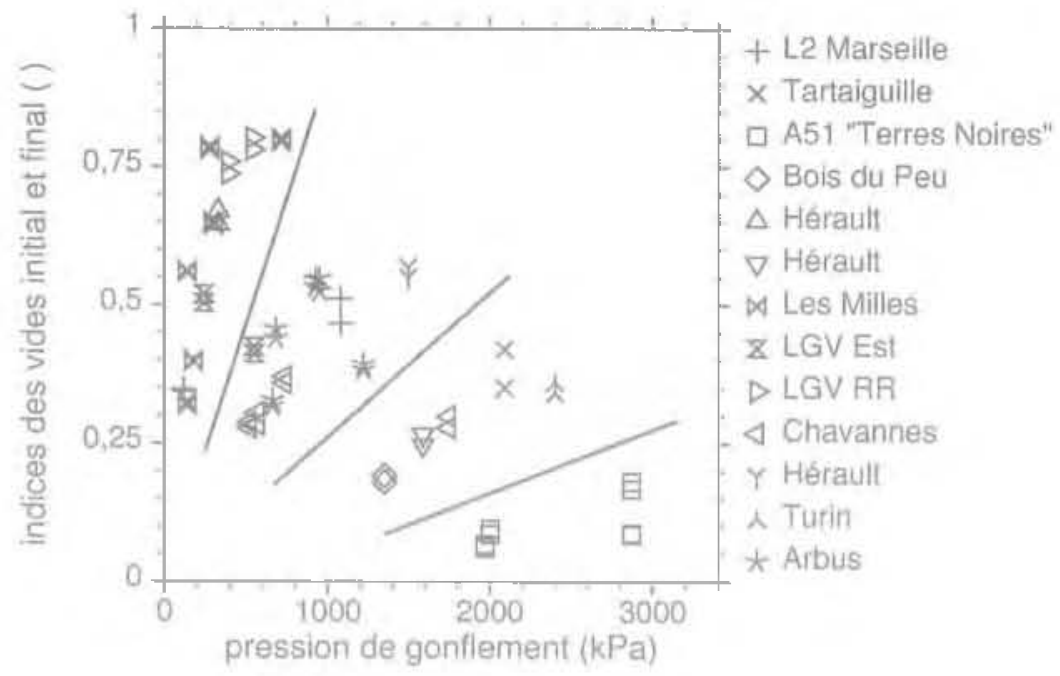

nG. 14 Pressions de gonflement et variations de l'indice des vides au cours du gonflement pour treize marnes. Swelling pressures and variations of void ratio during swelling for thirteen marls.

5.9

\section{Dynamique de retrait/gonflement}

Les graphiques de la figure 15 associent l'indice des vides initial $\mathrm{e}_{\mathrm{n}}$ aux variations $\Delta \mathrm{e} \mathrm{d}^{\prime}$ indice des vides de gonflement ou de retrait mesurées au laboratoire. Le cadre de cette comparaison doit être précisé, car les variations de volume de retrait et de gonflement ne sont pas strictement comparables de par la nature différente des essais. Dans les essais de gonflement vedométriques, les variations de volume sont contrôlées par la charge (et les effets tridimensionnels), contrairement aux essais de retrait où les variations de volume de dessiccation en atmosphère ambỉante ne dépendent que du sol et de la dimension des éprouvettes. Aussi, pour le gonflement, la variation de volume sera égale a $\Delta \mathrm{e}=\Delta \mathrm{e}_{1, y}$ C'est à dire l'amplitude du gonflement à partír de $10 \mathrm{kPa}$ définie plus haut. Pour le retrait, $\Delta$ e représente la variation totale de volume mesurée entre le début et la fin de l'essai. Ce choix arbitraire n'entache pas les conclusions qui seront tirées des graphiques.

Les matériaux sont des matnes et des argiles testées dans leur état naturel, des sols compactés ou des sols déstructurés saturés lâches. Deux points supplémentaires de retrait ont été ajoutés sur lá fịure 15a, représentant une montmomillonite (m) et une kaolinite (k) déstructurées et saturées (Briaud et Zhang. 2004). Ces graphiques prolongent la notion évoquée précédemment selon laquelle les pressions de gonflement les plus fortes ne peuvent être attendues gue dans les terrains les plus compacts. Les figures $15 \mathrm{a}$ et $15 \mathrm{~b}$ montrent que les amplitudes totales des déformations de retrait et de gonflement semblent d"autant plus grandes que l'indice des vides initial est grand. Cette amplitude pourrait être qualifiée de c dynamique de retrait/gonflement $n$. Le terme a dynamique os est employé ici au sens d'une amplitude. Mais il ne ressort pas une règle unique de variation de $\Delta e$ avec $\mathrm{e}_{\text {devant }}$ la variété des réponses des dirférents terrains dans différents états et leur propre hétérogénéité. Par contre, il apparait plus nettement gue la plus grande part des variations de volume des sols lậches relève du retrait, tandis que la plus grande part des variations de volume des terrains compacts relève du gonflement. Ainsi, les sols déstructurés salurés à forte teneur en eau ne gonflent pas et les terrains compacts ne se rétractent pas. Des exemples ont été donnés sur la figure 13.

La droite (1) de pente -1 indiquée sur les graphiques délimite le domatne des retraits possibles, c'est à dire $-e_{0}<\Delta e<0$. Les trois autres courbes $(2,3$ et 4 ) expriment une relation entre e et $\Delta$ e telle que :

$$
\Delta \mathrm{e}=\mathrm{e}_{\mathrm{x}}\left[1-\exp \left(-\mathrm{e}_{\mathrm{g}} / \mathrm{b}\right)\right\}-\mathrm{e}_{\mathrm{o}}
$$

avec $\mathrm{b}=-\mathrm{e}_{\mathrm{on}} / \ln \left(1-\mathrm{e}_{\mathrm{on}} / \mathrm{e}_{\mathrm{k}}\right)$ et $\mathrm{e}_{\mathrm{k}}$ et $\mathrm{e}_{\text {on }}$ des paramètres. La variation de volume de retrait-gonflement est nulle $(\Delta \mathrm{e}=0)$ si $\mathrm{e}_{\mathrm{o}}=0$ ou $\mathrm{e}_{\mathrm{a}}=\mathrm{e}_{\mathrm{on}}$. Le paranètre $\mathrm{e}_{\text {on }}$ peut être considéré comme un paramètre de structure, car le sol présente une dynamique de gonflement si $0<e_{0}<e_{m}$ et une dynamique de retrait si $\mathrm{e}_{\text {an }}<\mathrm{e}_{\mathrm{o}}<\mathrm{e}_{\mathrm{k}}$. Le parametre e dépend de la nature du sol et son mode de formation. Les trois courbes de la figure 15 sont calculées avec les couples $\left(e_{\mathrm{k}}, e_{\mathrm{an}}\right)$ égaux à $(0,4 ; 0,04),(1,6 ; 0,6)$ et $(3$; 2) respectivement.

Quelques matériaux particuliers sont mis en exergue sur la figure 15b, parmi l'ensemble des données de la figure 15a. Les molasses du mont Sion se regroupent du côté des terrains gonflants et des fortes compacités. Toutefois, les molasses les pius expansives ne sont pas les plus compactes (figure 11). Largile des Milles, qui est peu compacte et hétërogène, présente des déformations de retrait plus grandes que celles de gonflement. Une tendance analogue s'observe avec le limon de Goderville compacté en différents points d'état (Serratrice, 2007),

Il rie doit pas être perdu de vue que cette perception globale du comportement des différents matériaux dans différents états résulte d'une approche macroscopique au laboratoire qui est transcrite ici par des paramètres tels que $K, \sigma_{\text {ag }}$ ou $\Delta \mathrm{e}$. En pratique, la dynamique de retrait/gonflement dépend de nombreux facteurs comme la nature du sol, sa composition minéralogique, sa structure pour les terruins Lestés dans leur état naturel, son anisotropie, le mode de préparation et de densification au laboratoire pour les sols compactés ou les sols déstructurés. Néanmoins, l'état de compacité initial du matériau, traduit ici par $e_{o}$ détermine pour 

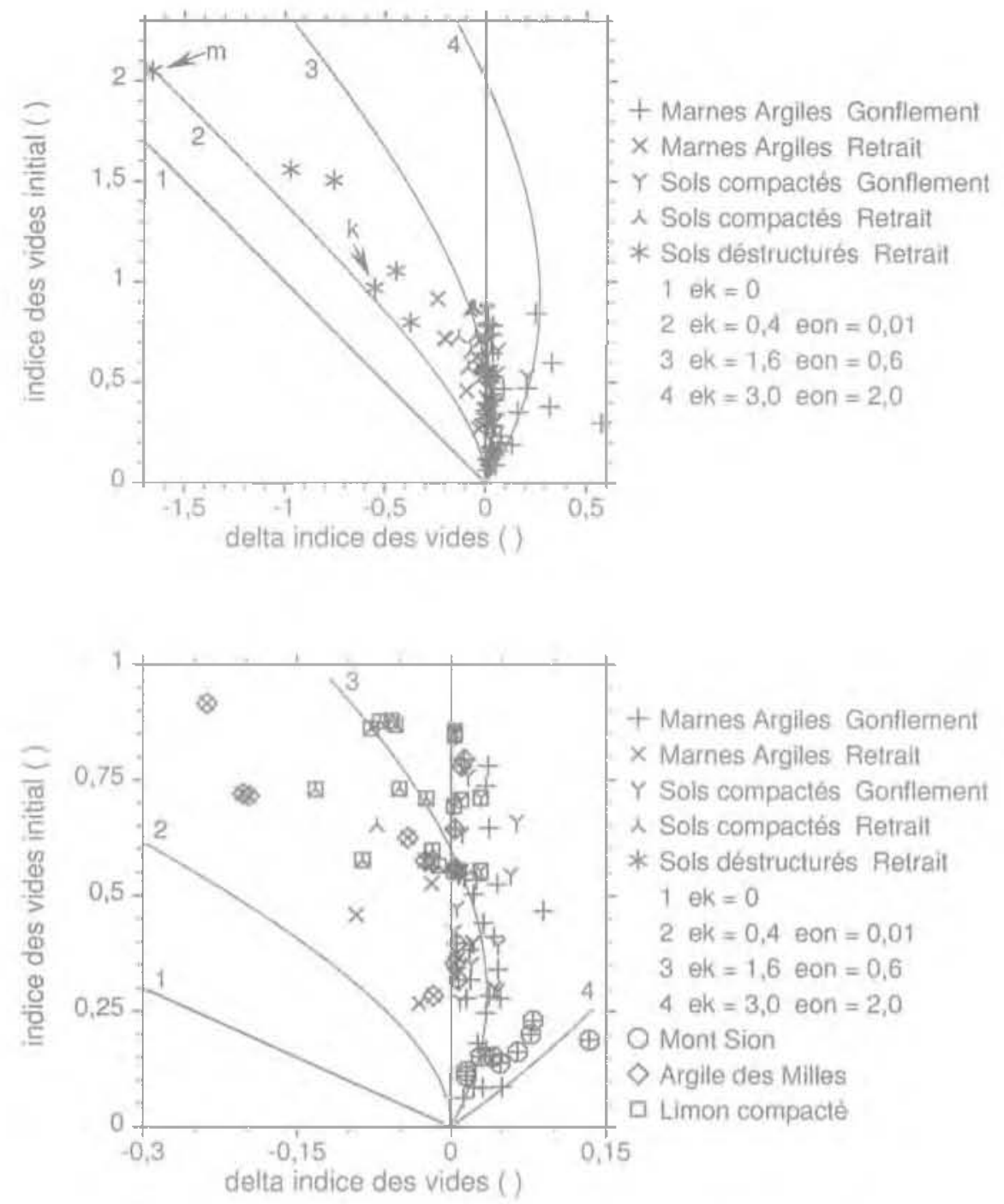

me is Variations de volume de retrait/gonflement.

a) Ensemble des données ; b) Matériaux particuliers.

Shrink-sweil volume chances

a) All data : b) Particular soils.

une large part cette dynamigue. Les courbes tracées sur les graphiques de la fígure 15 suggèrent que, malgrể la variabilité des compacités et des compositions minéralogiques des terrairs d'un même site, chaque formation semble posséder une seule dynamique de retrait/gonflement. Mais, en pratique, il n'existe pas de terrains dont l'hétérogénéité provjenne de la seuie variabilité des compacités.

\section{6}

\section{Conclusion}

Cette présentation de quelques aspects expérimentaux du gonflement et du retrait vus au travers d'exemples d'essais de laboratoire ne fait qu'évoquer des questions connues depuis longtemps, tant sur le plan de la pratique des essais que de la compréhension des phénomènes et la transposition de ces données au comportement des ouvrages.

La cinétique du gonflement est très lente pour les terrains compacts testés dans leur état naturel. Elle prend souvent l'allure de courbes de consolidation inversées, avec une phase de a gonflement secon daire o linéaire en logarithme du temps sur de longues périodes. Le gonflement (et le retrait) sont des mécanismes tridimensionnels. Les essais oedométriques $\mathrm{K}$ (mesure de la contrainte radiale) font apparaître de trés forts états de contrainte d'extension pouvant conduire à la rupture du sol dans les bagues oedométriques, Des déformations plastiques se superposent alors aux déformations de gonflement, ce qui permet d'avancer que lés potentiels de gonflement peuvent être surestimés dans bien des cas. L'utilisation de bagues oedométriques déformables confirme cette tendance. Ces effets tridimensionnels peuvent expliquer aussi pourquoi les pressions de gonflement oedométriques dépendent du protocole d'essai. En présence de terrains dotés d'un fort potentiel de gonflement, il paraît nécessaire de pratiquer des essais à haute pression susceptibles de dépasser la pression de gonflement et s'assurer alors de la nullité des déformations. Enfin, le gonflement d'un sol se traduit par une dégradation de ses propriétés mécaniques, qui peut être vue comme le résultat d'un mécanisme d'altération à l'échelle du temps des essais de laboratoire. 
L'amplitude des déformations qu'in sol est susceptible de développer, pendant l'un ou l'autre des dew mécanismes de retrait/gonflement, a été qualifiée de "dynamique de retrait/gonflement ». Si l'approche macroscopique pratiquée au laboratoire et illustrée ici au Lravers de différents exemples ne permet pas d'expliquer en détail les mécanismes observés, il ressort néanmoins que cette dynamique dépend de la compacité initiale du sol, en première approximation. En termes de potentiel de déformation et pour les matériaux sensibles aux variations hydriques, un sol lâche produira préférentiellement une dynamique de retrait, ałors qu'un matériau compact produira plutôt une dynamique de gonflement. Dans ce sens, les pressions de gonflement les plus élevées sont à attendre de la part des sols les plus compacts. Cela sous-tend l'idée que, en termes d'effets de structure, l'espace poreux disporible dans le sol et les liaisons d'induration associées, jouent vraisemblablement un rôle dans les variatons de volume observées à une échelle macroscopique. Ces différentes observations tirées des essais de laboratoire ne sont pas sarns analogie avec les observations pratiquées sur les ouvrages.

\section{Bibliographie}

AFNOR - Norme XP P 94060-2, Détermination effective de la limite de retrait sur un préièvernent non remanié, décembre $1997,10 \mathrm{p}$.

AFNOR - Norme XP P 94-091, Essaj de gonflement à l'oedomètre. Détermination des defformations par chargement de plusieurs éprouvettes, décembre $1995,13 \mathrm{p}$

Bigot G., Zerhouni M.I. - Retrait, gorflement et tassement des sols fins. Bult. des Labo. des P et Ch., 229, 2000, p. 105-114.

Briaud J.-L, Zhang $X$, - Water content method for movement prediction of swell shrink soils. Symp. Int. Fondsup, Paris 2004, vol. 2. p. 353-372.

Gaudin B., Serratrice J.-F. - Etude en laboratoite du comportement mécanique d"une molasse. 2nd Int. Symo. on Flard Soils - Soft Rocks, Naples, 1998 , tone 1, p. 173-181.

Guiliaume A., Jeanette D., Pequet H., Serratrice J.-F. - Géotechnique de la formation d'Esna et conservation de la tombe de Ramsès II (Vallée des Rois, Lougsor, Egypte]. Revue d'Archéométrie, $n^{\circ}$ 25, 2001. p. 113-123.
ISRM. - Suggested methods for laboratory testing of argillaceous swelling rocks. Int. J. Fock Mech. Min. Sci. \& Geomech. Abstr, vol. 26, n $n^{\circ} 5$, p. 415426.

Leroux A. (1972) - Caractérisation mécarifques des roches argileuses en relation avec leur texture. Bull. liaison Labo. P. et Ch. 61, 1972, p. 155-178.

Mieussens C. - Détermination de la sensibilité des sois aux variations de teneur en eau en laboratoire. Essais oedométriques sur les sols compactés. Projet de méthode d"essaj des LPC. LPC de Toulouse, $199 \mathrm{~s}$.

Pejon O.J., Le Roux A., Guignard D. - Comportement à l'eau des roches argilo-marneuses, suivi du gonflement, importance de la minéralogie et des textures. Bull AIEG, Paris, 55, avit 1997 ; p. $105-119$.

Serratrice J.-F, Flavigny E. - Mesure en laboratoire du coefficient $\mathrm{K}$ d'une marne Ist Int Symp, on Hard Soils - Soft Rocks, Athènes, 1993, vol. 1. p. $787-793$

Serratrice J.F. - Comportement d'une argile compactée. Bull haison Labo. P. et $C h, 200,1995$, p. $13-23$

Serratrice J.F. - Comportement d'une craie compactée. Colloquím Mundanum a Craies et Schistes v, GBMR, Bruxelles, 1995, p. 1.1.711.1.1.80.

Serratrice J.-F. - Comportement d"ur limon compacté. Bull des labo. P, et Ch. 2007, à paraître.

Serratrice J.-F, Soyez B. - Les essais de gonflement. Bwll. liasson Labo. P. et Ch, 204, 1996, p. 65-85

Sridharan A., Reo A S. Sivepullaiah PV, - Swelling pressure of clays. Geotech. Tosting J., vol. 9. $\pi^{\circ}{ }^{\circ}$ 1, 1986, p. 24-33.

Wudal T. - Etude en laboratoire du gonflement des sols : mise au point d'un oedométre flexible et étude du gonflement tridimensionnel. Thèse de l'Unjversitê des sciences et technalogies de Lille, 2001, $128 \mathrm{p}$.

Windal T, Shahrour I, Magnan J.-P., Serratrice J.-F - Etude du gonflement des sols pour les projets de tunnels. Revue francaise de géotechnique, $\mathrm{n}^{\circ} 100,2002$, p. 91-99. 